\title{
Pre-hospital management of severe traumatic brain injury- compliance with guidelines
}

\author{
Rebecka M Rubenson Wahlin", Johannes Gustavsson, Maaret K Castrén \\ From London Trauma Conference 2013 \\ London, UK. 10-13 December 2013
}

\section{Background}

Traumatic brain injury (TBI) is a major cause of death and disability. Guidelines for pre-hospital management of severe TBI have been published but research has shown that pre-hospital management often differs from that recommended in guidelines.

\section{Aim}

To assess compliance with guidelines in the pre-hospital management of severe TBI in Stockholm, in assessment and/or treatment of airway, ventilation, oxygenation, blood pressure, fluid resuscitation, GCS, and pupil properties.

\section{Method}

The study was a cross sectional observational study based on data from trauma registry and ambulance records. Included patients $(\mathrm{n}=101)$ were adult (age $>15$ years), with Injury Severity Score $>9$ and GCS 3-8 (severe TBI caused by blunt trauma), transported by ambulance or helicopter and admitted to the trauma center at Karolinska University Hospital in Stockholm between January $1^{\text {st }}$ and December $31^{\text {st }}, 2008$. TBI were confirmed by computer tomography and with a corresponding ICD-10 diagnosis (s06.0-06.91). The pre-hospital management considered compliant with guidelines only if the assessment or the treatment was documented in the ambulance journal. A $65 \%$ criterion was used as a lower limit for acceptable compliance.

\section{Results}

Compliance with guidelines was; oxygenation assessment $90.1 \%$, hypoxia treatment $100.0 \%$, blood pressure assessment $88.1 \%$, hypotension treatment $90.0 \%$,

Department of Clinical Science and Education, Södersjukhuset, Karolinska Institute, Stockholm, Sweden 